\title{
Preface
}

CogInfoCom is an interdisciplinary research field that has emerged as a synergy between infocommunications and the cognitive sciences.

One of the key concepts behind CogInfoCom is that humans and ICT are becoming entangled at various levels, as a result of which new forms of blended cognitive capabilities are appearing. These new capabilities - not separable into purely natural (i.e., human), or purely artificial components - are targeted towards in theoretical investigations and engineering applications.

This special issue presents various new results on this scientific disciplina in the followin papers:

\section{1) Whispered Speech Recognition using Hidden Markov Models and Support Vector Machines}

This paper presents an analysis in recognition of whisper using 2 machinelearning techniques: Hidden Markov Models (HMM) and Support Vector Machines (SVM). The experiments in paper are conducted in both Speaker Dependent (SD) and Speaker Independent (SI) fashion for Whi-Spe speech database. The best neutral-trained whisper recognition accuracy in SD fashion $(83.36 \%)$ is obtained in SVM framework. At the same time, HMM-based recognition gave the highest recognition accuracy in SI fashion (87.42\%). The results in recognition of neutral speech are given as well.

\section{2) Evaluation of Cognitive Processes using Synthesized Words: Screening of Hearing and Global Speech Perception}

This study focuses on children's cognitive capability within the framework of cognitive infocommunication. The paper shows that the great majority of children were confirmed to have good hearing (about 95\%), while some children had a previously unknown hearing impairment. More than $30 \%$ of all children encountered speech perception deficit despite good hearing. Digital technology including speech synthesis has reshaped both speech science and its cognitive connections to get closer to a proper interpretation of the mechanisms analyzed.

\section{3) Assessing the Children's Receptivity to the Robot MARKO}

This paper presents an experimental assessment of the children's receptivity to the human-like conversational robot MARKO. It reports on a production of a corpus that comprises of recordings of interaction between children, with cerebral palsy and similar movement disorders, and MARKO, in realistic therapeutic settings. The evaluation of the corpus showed that the positive effects go beyond social triggering - the children not only positively responded to MARKO, but also experienced increased motivation and engagement in therapy. 


\section{4) A Content-Analysis Approach for Exploring Usability Problems in a Collaborative Virtual Environment}

This paper introduces a framework for the usability evaluation of collaborative 3D virtual environments based on a large-scale usability study of a mixed-modality collaborative VR system. Twelve different usability problems were identified, and based on the causes of the problems, we grouped them into three main categories: VR environment-, device interaction-, and task-specific problems. The framework can be used to guide the usability evaluation of collaborative VR environments.

\section{5) Gain-Scheduling Control Solutions for Magnetic Levitation Systems}

The paper presents three Gain-Scheduling Control (GS-C) design procedures starting with classical Proportional-Integral (PI) controllers, resulting in PI-GS-C structures for positioning control of a Magnetic Levitation System (MLS) with two electromagnets laboratory equipment.

\section{6) Corrective Focus Detection in Italian Speech Using Neural Networks}

This paper develops an Artificial Cognitive System (ACS) based on Recurrent Neural Networks that analyzes suitable features of the audio channel in order to automatically identify the Corrective Focus on speech signals. Two different approaches to build the ACS have been developed. The experimental evaluation over an Italian Corpus has shown the ability of the Artificial Cognitive System to identify the focus in the speaker IUs. The addressed problem is a good example of synergies between Humans and Artificial Cognitive Systems.

\section{7) Relevance \& Assessment: Cognitively Motivated Approach toward Assessor-Centric Query-Topic Relevance Model}

This paper intends to introduce a novel model for query-topic relevance assessment from assessor and cognitive point of view in the sense that relevance is a multidimensional cognitive and dynamic conception. The focus of this presentation is concentrated on modeling the concept "Query Associative Vocabulary of Relevance" to emphasize the value of integrating intuitive, descriptive, multi-valued assessment, and agreement in the process of creating a query-topic relevance data. As this model differentiates between different types of query-topics and levels of relevance, it provides a facility to enhance the quality of relevance data by re-evaluating the resulted associative vocabulary at each cycle of refinement.

\section{8) Cognitive Aspects of Spatial Orientation}

This paper focuses on cognitive aspects of spatial mental modeling. We examine possibilities for merging methods for sensing and modeling of cognitive capabilities and cognitive styles with the concept of cognitive infocommunications. Related aspects of cognitive psychology, the theory of 
senses, sensory substitution, and mental modeling are discussed. The paper illustrates practical impact of emerging CogInfoCom methods on people with special needs, in particular, those with vision impairment.

\section{9) The Centrencephalic Space of Functional Integration: a Model for Complex Intelligent Systems}

This paper aims to show how the success or failure of a balanced man-machine co-evolution will also depend on some answers to fundamental scientific questions that have remained unexplored, such as consciousness and decision-making, creativity, but above all to the adaptive factor that more radically sustained and pushed the evolution beyond the constraints of our genetic code:improvisation. This entanglement of neuronal matrices could be at the origin of an intermodal communication consists of a stream of semantic phenomena, mental images and more, tuned thanks to "pattern recognition" in centrencephalic space of functional integration - thus explaining "remote spectrum actions" at the base of primary adaptive unconscious and experiences life.

\section{0) An Interactive Haptic System for Experiencing Traditional Archery}

This paper presents a first attempt to create a CogInfoCom channel through which a Virtual Reality (VR) system communicates with a natural cognitive system (prototype and physical experimental system) in a way that improves human cognitive abilities to understand the way an ancient bow works and the sensations it exerts on the human body. This study proposes an immersive VR simulator for recreating the experience of shooting with 3 types of old bows, based on a customized haptic interface.

\section{1) Use of Augmented Reality in Learning}

Augmented reality offers great solutions in learning because most of high school students are familiar with them. In this study authors first introduce the augmented reality and a specific application, Pokémon Go, then demonstrate the use of AR in education and finally present a survey conducted among students of a higher education in Hungary.

\section{2) Educational Context of Mathability}

Mathability in its definition refers to cognitive infocommunication and combines machine and human cognitive capabilities essential for mathematics. In the paper educational aspects of the notion are considered. A new proposal of learning outcomes taxonomy is presented.

\section{3) Urban Scaling of Football Followership on Twitter}

This paper analyzes followers of prominent footballs clubs on Twitter by obtaining their home locations. We then measure how city size is connected to the 
number of followers using the theory of urban scaling. The results show that the scaling exponents of club followers depend on the income of a country. These findings could be used to understand the structure and potential growth areas of global football audiences.

\section{Péter Baranyi}

Special Session Guest Editor 